\title{
UPGRADES TO THE FERMILAB NUMI BEAMLINE*
}

\author{
FERMILAB-CONF-07-629-AD \\ Michael A. Martens", Sam Childress, Nancy Grossman, Patrick Hurh, James Hylen, Alberto \\ Marchionni, Elaine McCluskey, Craig Damon Moore, Robert Reilly, Salman Tariq, Alan \\ Wehmann, Karlton Williams, Robert Miles Zwaska, Fermilab, Batavia, Illinois.
}

\begin{abstract}
The NuMI beamline at Fermilab has been delivering high-intensity muon neutrino beams to the MINOS experiment since the spring of 2005 . A total of $3.4 \times 10^{20}$ protons has been delivered to the NuMI target and a maximum beam power of $320 \mathrm{~kW}$ has been achieved. An upgrade of the NuMI facility increasing the beam power capability to $700 \mathrm{~kW}$ is planned as part of the NOvA experiment. The plans for this upgrade are presented and the possibility of upgrading the NuMI beamline to handle 1.2 MW is considered.
\end{abstract}

\section{INTRODUCTION}

The NuMI beamline uses $120 \mathrm{GeV}$ protons from the Main Injector (MI) to produce a high intensity $v_{\mu}$ beam. The protons strike a graphite target producing pions (and some kaons) which are then focused by two pulsed magnetic horns. The pions travel through a 675 meter long decay pipe allowing time for the pions to decay; $\pi^{-}$ $\rightarrow \mu^{-}+v_{\mu}$. A hadron absorber at the end of the decay pipe intercepts any remaining hadrons. The muons are absorbed by a layer of rock downstream of the hadron absorber. This leaves a $v_{\mu}$ beam angled downward by 58 mrad pointing at the MINOS near detector at Fermilab and the MINOS far detector $735 \mathrm{~km}$ away in Soudan, Minnesota [1].

During normal operations the $120 \mathrm{GeV}$ MI beam is shared between NuMI and antiproton production for Collider Run II. This leaves $\sim 200 \mathrm{~kW}$ of beam power available for the MINOS experiment. During periods without antiproton production all the MI beam is available for NuMI and the beam power has reached $320 \mathrm{~kW}$. More information on the status of NuMI can be found in $[2,3]$.

Future experiments utilizing the NuMI facility are the MINERvA neutrino scattering experiment and the NOvA experiment to search for the appearance of the electron neutrino. NuMI upgrades for these experiments are best described by three different upgrades: The ongoing Fermilab Proton Plan [4], the Accelerator and NuMI Upgrades (ANU) for the NOvA [5] experiment, and the conceptual Super-NuMI (SNuMI.) [6]. Beam parameters for these upgrades are shown in Table 1. This paper focuses on the NuMI beamline and information on the upgrades to the accelerators can be found in [7].

The Proton Plan is a set of ongoing improvements to the Fermilab accelerator complex aimed at increasing the throughput of the Booster. When completed in early 2008 NuMI will be operating at a beam power of $320 \mathrm{~kW}$ (or as

\footnotetext{
*Supported by the U.S. DOE contract No. DE-AC02-07CH11359

\#martens@fnal.anl.gov
}

much as $400 \mathrm{~kW}$ if antiproton production is not needed.)

The ANU will increase the NuMI beam power to 700 $\mathrm{kW}$ by converting the Fermilab Recycler Ring from a antiproton storage ring into a pre-injector for the Main Injector. The ANU will be complete in $\sim 2011$ as the NOvA experiment is beginning to come on-line.

SNuMI (Super-NuMI) is only conceptual and would use Antiproton Accumulator to momentum stack protons from the Booster and deliver 1.2 MW of beam power to the NuMI target.

Table 1: Beam Parameters for various NuMI Upgrades

\begin{tabular}{|c|c|c|c|c|}
\hline & $\begin{array}{c}\text { Present } \\
\text { Conditions }\end{array}$ & $\begin{array}{l}\text { Proton } \\
\text { Plan }\end{array}$ & NOvA & SNuMI \\
\hline $\begin{array}{c}\text { Booster intensity } \\
\text { (protons/batch) }\end{array}$ & $4.8 \times 10^{12}$ & $4.3 \times 10^{12}$ & $4.3 \times 10^{12}$ & $4.7 \times 10^{12}$ \\
\hline MI cycle time (s) & 2.4 & 2.2 & 1.333 & 1.333 \\
\hline $\begin{array}{l}\text { MI Intensity to } \\
\text { NuMI (ppp) }\end{array}$ & $2.4 \times 10^{13}$ & $3.7 \times 10^{13}$ & $4.9 \times 10^{13}$ & $8.3 \times 10^{13}$ \\
\hline $\begin{array}{l}\text { Beam Spot } \\
\text { Size }(\mathrm{mm})\end{array}$ & 1.0 & 1.2 & 1.3 & $\sim 1.3-1.5$ \\
\hline $\begin{array}{l}\text { NuMI beam } \\
\text { power }(\mathrm{kW})\end{array}$ & $\begin{array}{c}200 \\
(320)^{*}\end{array}$ & $\begin{array}{c}320 \\
(400)^{*}\end{array}$ & 700 & 1200 \\
\hline $\mathrm{POT} / \mathrm{yr}$ to $\mathrm{NuMI}$ & $2 \times 10^{20}$ & $3 \times 10^{20}$ & $6 \times 10^{20}$ & $10 \times 10^{20}$ \\
\hline \multicolumn{5}{|c|}{$\begin{array}{l}\text { * NuMI is normally run concurrently with Collider Operations and a } \\
\text { portion of the Main Injector beam is used for antiproton production. In } \\
\text { parenthesis is the beam power available with NuMI only operations. }\end{array}$} \\
\hline
\end{tabular}

\section{PROTON PLAN}

The Proton Plan is an ongoing upgrade to increase the number of protons available from the Booster for use in collider operations and neutrino experiments. For NuMI, the most significant change will be the implementation of multi-batch slip stacking in the MI [8]. After the implementation of multi-batch slip stacking, the MI will be capable of accepting 11 Booster batches by injecting 6 Booster batches and slip stacking an additional 5 Booster batches on top of these. Two of the Booster batches are still used for antiproton production and the other 9 are used for NuMI. This will increase the proton intensity to NuMI by a factor of about $50 \%$. The NuMI beamline is designed to handle $400 \mathrm{~kW}$ of beam power, so no upgrades are needed for the NuMI beamline under the Proton Plan.

\section{ANU FOR NOVA}

There are three major categories of NuMI upgrades under ANU. The primary beamline, which delivers beam 
from the MI to the NuMI target, is upgraded to operate at the faster cycle time of 1.33 seconds. The target and horn optics are changed to optimize the neutrino beam energy spectrum for the NOvA experiment. The NuMI facility is upgraded to handle the increase in beam power.

\section{Primary Beamline}

The primary beamline is used to transfer $120 \mathrm{GeV}$ protons from the MI to the NuMI Target. As built it is capable of operating at a 1.87 cycle time and modification will give it the capability of 1.33 second cycle time.

The MI extraction kicker magnet does not need modification since the bunch spacing and beam energy do not change for the ANU. However, a new charging power supply is needed to meet the faster charging time required by the 1.33 second cycle and an upgraded Fluorinert-towater heat exchanger is needed to supply more cooling of the kicker magnets to compensate for the higher heat load caused by the faster repetition rate.

The most significant upgrade to the primary beamline is the replacement of five quadrupole magnets with limited cooling capacity. As built, NuMI uses $\sim 25$ year old quadrupole magnets (3Q120 magnets) which do not have internal cooling of the coils and are susceptible to failure under the higher heat loads generated by the faster cycle time. Five of these quadrupoles with the highest operating current have been selected for replacement with newer design quadrupole magnets (QQB magnets) which have internal coil cooling. The QQB magnets are already in use in the antiproton injection line from the MI to the Tevatron. When Collider Run II is complete and the antiproton injection line is no longer used the robust QQB magnets will be available as replacements in the primary beamline.

The large dipole power supplies of the primary beamline will be capable of a 1.33 second cycle time with only minor changes to the transformer taps. In addition, the measurement accuracy of the dipole current will be improved from $0.5 \%$ to $0.01 \%$ by implementing a BuLB regulation system. Since the dipole current is one of the inputs to the beam abort system, the improved accuracy will reduce the risk of damage from miss-steered beam.

\section{Medium Energy Neutrino Spectrum}

One of the significant changes to NuMI is the optimization of the neutrino energy spectrum for the NOvA experiment. The MINOS and MINERvA experiments run with the NuMI beamline in the low energy configuration which produces neutrinos in the $\sim 1$ $5 \mathrm{GeV}$ energy range at a location on the beam axis. (See Figure 1.) The NOvA experiment is optimized by locating the far detector $14 \mathrm{mrad}$ off-axis of the neutrino beam in order to narrow the width of the neutrino energy spectrum. Reconfiguring the NuMI beamline from the low energy to the medium energy optics narrows the spectrum further and increases the flux of neutrinos with energies near $2 \mathrm{GeV}$. The change in neutrino energy spectrum is accomplished by moving the target $135 \mathrm{~cm}$ further upstream and relocating the second focusing horn 13 meters further downstream.

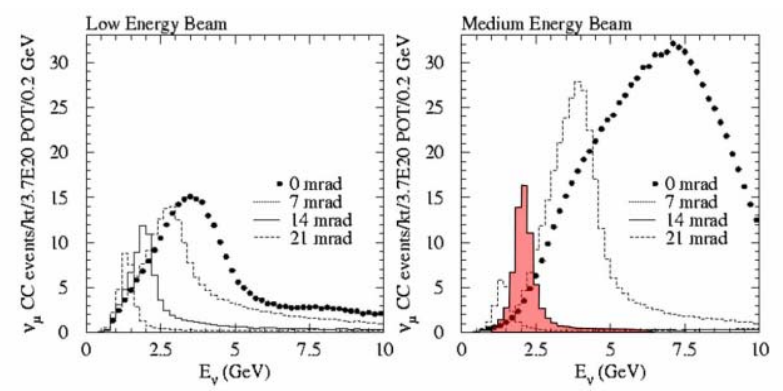

Figure 1: Charged Current $v_{\mu}$ event rates under a nooscillation hypothesis at a distance of $800 \mathrm{~km}$ from Fermilab at various transverse locations for the NuMI low energy beam configuration (left) and medium energy beam configuration (right). The neutrino spectrum at the NOvA far detector is highlighted in red.

Relocating the second horn is conceptually simple but requires a significant amount of engineering effort. A space must be created in the target chase shielding at the new horn location and the gap left at the original horn location filled. Also the stripline that connects power supplies to the horns and water cooling connections must be extended by 13 meters. These tasks take place with a target chase that is radioactive from beam operations and in the target hall with limited space for maneuvering

\section{Higher Beam Power}

Many of the upgrades to NuMI are related to the larger heat load resulting from the increase in beam power from $400 \mathrm{~kW}$ to $700 \mathrm{~kW}$. One important aspect is the understanding of the target pile heat loads and temperature profile. The target chase is cooled by circulating chilled air around and through the target chase. Upgrades already planned for the fall of 2007 will increase the chiller capacity. Modeling of the target chase energy deposition and heat flow shows that the temperature of the target pile at its hottest location would reach $113{ }^{\circ} \mathrm{C}$ while operating at the $700 \mathrm{~kW}$ of beam power for the NOvA experiment.

A new target will be designed and built for the NOvA experiment. The target presently in use is constrained to a diameter of $30 \mathrm{~mm}$ since it must fit within the radius of the horn inner conductor. For NOvA the target is located further upstream and the target casing can be made larger since it doesn't need to fit within the horn. This leads to the design choice of graphite plates clamped between two water cooled aluminum plates as shown in Figure 2 and described in [9]. The graphite plates are $6.4 \mathrm{~mm}$ wide, the target is $135 \mathrm{~cm}$ long with 12 plates. An analysis of the thermal stresses on the target graphite has been done and with a beam spot size of $1.3 \mathrm{~mm}$ rms in both transverse planes the mechanical safety factor is 1.7 . The location of the water cooling lines further away from the beam reduces the beam energy deposition in the water eliminates problems with the water hammer effect. 


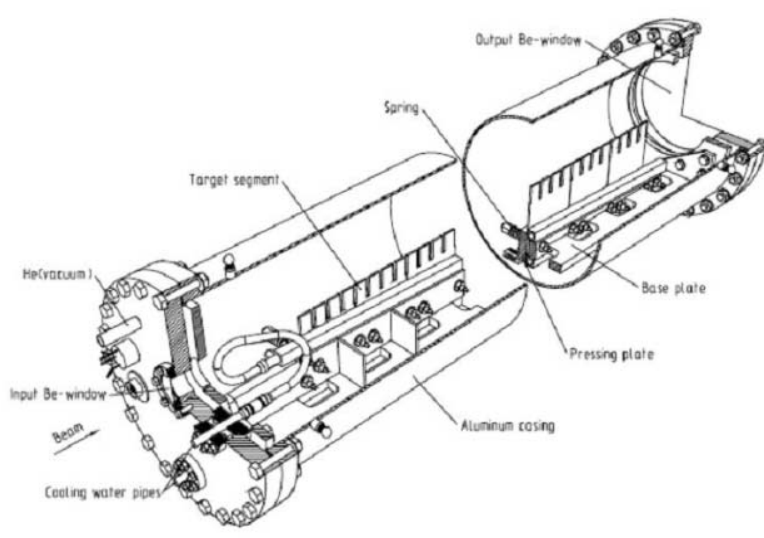

Figure 2: Schematic of the medium energy target.

Only minor modifications are needed to the design of the magnetic focusing horns to handle the increase in beam power. The horns are cooled with internal water sprays on the inner and outer conductors. To reduce the energy deposition in the horns, the thickness of the outer conductor will be reduced from 1 inch to $\sim 5 / 8$ inch. This reduces the amount of material intercepting beam and still maintains the structural integrity of the horn. Additional cooling may be needed for the striplines in the vicinity of the horn.

The decay pipe, decay pipe window, and hadron absorber were also analyzed for the operations at the higher beam power. These elements of the NuMI beamline are capable of handling the higher beam power if the cooling capacity is increased.

Recent operational experience has also led to a change in philosophy regarding the replacement and repair of target hall components. There have been several instances where repairs of the horns were possible by using a work cell to shield personnel from the radiation. Given the expense, time, and manpower needed to build spare targets and horns, it is more efficient if they can be repaired safely. This has led to plans for upgrades to the work cell to include more radiation shielding and the use of tele-manipulators for remote handling.

\section{SNUMI}

Upgrades beyond the $700 \mathrm{~kW}$ planned for NOvA are conceptualized as part of a SNuMI. By using the Fermilab antiproton accumulator to momentum stack batches of Booster beam the proton intensity could reach $8.3 \times 10^{13}$ corresponding to $1.2 \mathrm{MW}$ of beam power at a 1.33 second cycle time. The two major challenges for the NuMI beamline under these conditions are the instantaneous beam intensity delivered to the target and the cooling upgrades needed to handle the increased power deposition.

A conceptual design of target capable of handling up to $2 \mathrm{MW}$ has been studied [10]. One possibility is a water cooled target with a $15 \mathrm{~mm}$ cylindrical graphite core encapsulated with pre-stress into a stainless steel or aluminum thin-walled pipe. Shock absorption in the cooling system might be accomplished with two-phase water flow realized either by loading of the cooling water with He or Ar bubbles (10-15\% of volume) or by the use of boiling condition in the cooling channel. Compared to the fin design, the cylindrical target would reduce the number of neutrinos per proton on target by about $10 \%$.

Cooling of the target pile is another challenge. Currently the target pile is cooled by flowing chilled air past the outside of the target pile and down the center of the target pile. This is sufficient to handle the $280 \mathrm{~kW}$ of power deposited in the target pile for NOvA operations, but requires $12 \mathrm{~m}^{3} / \mathrm{s}$ of chilled air flow which reaches speeds of $8 \mathrm{~m} / \mathrm{s}$ at some locations. This is near the limit of what is achievable with air cooling. Under SNuMI it would be necessary add water cooling to the target pile. Space limitations and the radioactivity of the target pile will make this challenging especially since it will be necessary to remotely install water cooling. However, about $80 \%$ of the beam energy is deposited within the innermost two inches of the target pile so that relatively thin water cooled plates could intercept a large fraction of the total energy deposited in the target chase.

Cooling of the first horn is also an issue for NuMI. In the present design the outer conductor of the horn is cooled by a film of water sprayed on the inside surface. Even with the thinner conductor planned for the NOvA upgrade it won't be possible to remove enough heat using this method and additional cooling would have to be added to the horn.

The survivability of the decay pipe and decay pipe window has been examined under SNuMI conditions and it has been determined that these elements are fine under normal operating conditions. The elements can also survive up to 10 consecutive accidental beam pulses which could hit the window directly. Beam permit inputs are needed to monitor this.

\section{CONCLUSIONS}

Upgrades are planned for the NuMI beamline as part of the NOvA project which will increase the capacity to 700 $\mathrm{kW}$. Further upgrades would be needed to operate the NuMI beamline at 1.2 MW as envisioned under the SNuMI scenario.

\section{REFERENCES}

[1] J. Hylen, et. al., Fermilab-TM-2018, Sept., 1997.

[2] S. Kopp, Proc. Part. Accel. Conf. (2005), p. 1.

[3] R.M. Zwaska, et. al., Proceedings of this conference.

[4] "The Fermilab Proton Plan," www-accel-proj.fnal.gov/Proton_Plan/index.shtml

[5] "The NOvA proposal", www-nova.fnal.gov.

[6] "SNuMI Conceptual Design Report," Nov 2006.

[7] I. Kourbanis, Proceedings of this conference.

[8] K. Seiya, et. al., Proceedings of this conference.

[9] V. Garkusha, et. al., Report from IHEP, Protvino, Nov 27, 2006.

[10] V. Garkusha, et. al., Report from IHEP, Protvino, July 30, 2005. 